\title{
Les niveaux de référence diagnostiques en France: une perception contrastée face à un dispositif perfectible mais efficace
}

\author{
P. Roch ${ }^{1, *}$, D. Célier ${ }^{1}$, C. Dessaud ${ }^{2}$ et C. Etard ${ }^{1}$ \\ 1 IRSN, Unité d'expertise en radioprotection médicale, PSE-SANTE/SER/UEM, 31 avenue de la division Leclerc, \\ 92260 Fontenay-aux-Roses, France. \\ 2 CERAP, 4 rue René Razel, Domaine Technologique de Saclay, 91400 Saclay, France.
}

Reçu le 12 octobre 2017 / Accepté le 5 février 2018

\begin{abstract}
Résumé - Les niveaux de référence diagnostiques (NRD), introduits par la CIPR au début des années 1990, sont destinés à aider les professionnels de l'imagerie médicale à évaluer leurs pratiques et à optimiser les doses délivrées aux patients. Établis en France depuis 2004, initialement sur la base des recommandations européennes, les NRD sont mis à jour régulièrement par les autorités. Ces dernières s'appuient sur les résultats d'analyses nationales conduites par l'Institut de radioprotection et de sûreté nucléaire (IRSN), à qui a été confiée la responsabilité du recueil et de l'analyse des données de doses délivrées dans les établissements d'imagerie. Après cinq rapports d'analyse de l'IRSN, une révision de la réglementation en 2011, et à l'aube d'une nouvelle mise à jour en 2018, la France dispose d'un recul important sur les forces et les faiblesses du dispositif instauré en 2004. L'appropriation des NRD par les professionnels et l'adéquation des NRD avec la pratique clinique sont indiscutablement les deux pierres d'achoppement auxquelles sociétés savantes et institutions sont confrontées. Ce travail propose donc de dresser un bilan des réussites et des lacunes du dispositif des NRD français, et de présenter ses prochaines évolutions qui vont fournir aux professionnels un outil amélioré et actualisé pour les aider à optimiser les doses délivrées aux patients.
\end{abstract}

\begin{abstract}
Diagnostic reference levels in France: a contrasted perception opposed to a perfectible but efficient system. Introduced in the early 1990s' by ICRP, Diagnostic Reference Levels (DRL), are helpful tools for medical imaging professionals in order to optimise the radiation exposure of patients. Implemented in France in 2004, first from European DRLs, DRLs are periodically updated by regulatory authorities. Updates are based on the results of national surveys data carried out by IRSN which is in charge of the collection and the analyses of radiation doses delivered in French medical imaging departments. After five national assessments, a regulation update in 2011 and in the perspective of an update expected in 2018, this significant experience allows France to identify strengths and weaknesses of the system implemented in 2004. Professionals' involvement and consistency of DRLs with clinical practice are the cornerstones which challenge professionals' societies and authorities. The purpose of this work is to evaluate the balance between successes and failures of the French DRLs system and to introduce the future evolutions leading to give to the professionals an improved and up-to-date optimisation helping tool.
\end{abstract}

Keywords: medical imaging / patient radiation exposure / optimisation / diagnostic reference levels

\section{Introduction}

L'imagerie médicale occupe une place fondamentale dans la prise en charge des patients et représente la principale source d'exposition de la population aux rayonnements ionisants en

\footnotetext{
*Auteur de correspondance : patrice.roch@irsn.fr
}

France (Etard et al., 2012c; Etard et Aubert, 2014). Néanmoins, le niveau d'exposition résultant de l'immense majorité des procédures d'imagerie est considéré comme acceptable au regard des bénéfices qu'elles apportent. Au cours des dernières décennies, le nombre d'actes n'a cessé d'augmenter et les modalités d'imagerie ont évolué, conduisant nécessairement à considérer avec attention les risques potentiels liés à l'exposition répétée aux rayonnements 
ionisants. Un intérêt particulier doit notamment être porté à la scanographie («scanner») qui occupe progressivement la place la plus importante, sinon en nombre d'actes, mais en termes de dose délivrée aux patients.

En matière de radioprotection, tout individu est un patient potentiel et n'est pas soumis aux mêmes principes de radioprotection que les travailleurs ou le public. Justifier la prescription d'un examen et en optimiser la réalisation sont les fondements de la radioprotection des patients. Toute notion de limitation des expositions est proscrite dans ce domaine. En effet, limiter l'exposition des patients conduirait à constituer un obstacle aux soins si la situation d'un patient nécessitait la réalisation de nombreux examens ou traitements utilisant des rayonnements ionisants.

En matière d'optimisation en imagerie médicale, le Royaume-Uni fait figure de pionnier en ayant, dès les années 1980, initié un recueil de doses délivrées aux patients et encouragé les professionnels à optimiser leur pratique. Près de 30 ans plus tard, les doses délivrées aux patients au cours des examens ont globalement diminué de moitié dans ce pays (Hart et al., 2012; Shrimpton et al., 2014).

Au niveau international, dans les années 1990, les premières recommandations concernant les pratiques médicales ont émané de la Commission internationale de protection radiologique (CIPR), au nombre desquelles figure la mise en œuvre des Niveaux de Référence Diagnostiques (NRD) (ICRP, 1991, 1996). Élément primordial des exigences minimales de sûreté de l'Agence internationale de l'énergie atomique (AIEA), ils ont été intégrés à la réglementation européenne dès 1997, puis leur champ d'action a été renforcé dans la directive de 2013 (IAEA, 1996 rev. 2014; CE, 1997, 2014).

En France, lors de la transposition de la directive européenne de 1997, la mise en œuvre des NRD a été instituée en 2004 par voie réglementaire, confiant à l'Institut de radioprotection et de sûreté nucléaire (IRSN) la responsabilité d'organiser le recueil des données de doses auprès des établissements d'imagerie médicale et de restituer les résultats d'analyses à l'échelle nationale, en vue de la mise à jour périodique des NRD (JORF, 2004).

Durant ces treize années, l'IRSN a publié cinq bilans, dont certains ont conduit à mettre à jour la réglementation en 2011 (Talbot, 2008 ; Roch, 2010 ; JORF, 2012 ; Roch et Célier, 2012, 2014, 2016; Roch et Aubert, 2013). Ces rapports permettent à la fois de mesurer l'évolution de l'exposition des patients pour les établissements français d'imagerie médicale participant au recueil des NRD, mais aussi d'évaluer l'appropriation de la démarche d'optimisation au sein des établissements d'imagerie participants.

Cet article vise à dresser une synthèse de ces années de mise en œuvre de la démarche NRD en France, à la fois sous ses aspects vertueux en matière de radioprotection des patients, mais également dans sa dimension contraignante et partiale, telle que la ressentent parfois les professionnels de l'imagerie.

\section{Implication des professionnels: des bons et des mauvais élèves?}

Depuis 2004, tous les établissements réalisant des examens radiographiques, scanographiques et de médecine nucléaire doivent évaluer leurs pratiques, du point de vue dosimétrique, et transmettre leurs données à l'IRSN.
Si la participation est en progression depuis 2004, il apparaît néanmoins qu'elle est contrastée selon le domaine d'activité concerné (Fig. 1).

La médecine nucléaire et la scanographie ont atteint un niveau satisfaisant depuis 2013 avec respectivement près de 80 à $90 \%$ des établissements qui transmettent leurs données d'évaluations des doses chaque année à l'IRSN.

En radiologie, le taux de participation atteint à peine $30 \%$. Plusieurs explications peuvent être avancées.

En premier lieu, les activités de scanographie et médecine nucléaire sont soumises à autorisation par l'ASN, ce qui implique des formalités administratives et des inspections des installations au cours desquelles les relevés de données NRD sont examinés. Ce régime d'autorisation habitue également les professionnels à devoir répondre à des exigences réglementaires. Les installations de radiologie étant moins souvent inspectées par les autorités et soumises à un régime de déclaration, moins contraignant, les obligations réglementaires sont moins connues et appliquées (ASN, 2017a, 2017b, 2017c).

Ensuite, le recensement des installations de radiologie, du fait de l'aspect «volontaire» de la déclaration, est particulièrement difficile à établir. Le nombre de structures disposant d'installations de radiologie actuellement utilisé pour évaluer la participation (5100) repose sur une estimation ancienne, probablement devenue aujourd'hui obsolète, mais qu'il n'a malheureusement pas été possible d'actualiser car personne $-\mathrm{y}$ compris les autorités et organisations professionnelles comme a pu le constater la Cour des comptes en 2016- ne dispose à ce jour d'un recensement précis comparable à ceux de scanographie et de médecine nucléaire (Cour des comptes, 2016). L'IRSN conduit actuellement un travail destiné à estimer plus précisément le nombre de services disposant d'installations de radiographie en France. Les premiers résultats montrent que la surestimation du nombre de services pourrait atteindre $70 \%$ et conduirait donc à sous-estimer significativement la participation des services de radiologie (environ $50 \%$ en 2015 au lieu de $30 \%$ ) qui reste néanmoins nettement inférieure à celle de la scanographie et de la médecine nucléaire.

De plus, alors que les pratiques de la scanographie et de la médecine nucléaire s'adressent uniquement à des radiologues et à des médecins nucléaires, la radiologie est pratiquée par d'autres spécialités médicales (pneumologues, rhumatologues...) et dans des structures diverses (centres de santé, de médecine du travail...). L'activité d'imagerie dans ces structures n'étant pas le cœur de métier, la culture de la radioprotection, et des obligations afférentes, peut potentiellement y être mal appréhendée. Le nombre de structures de ce type peut cependant être considéré comme marginal par rapport aux établissements de radiologie.

Enfin, l'évaluation de la mise en œuvre d'une démarche d'optimisation sur la base du taux d'envoi de données à l'IRSN est un indicateur souvent discuté. Le fait d'envoyer des données à l'IRSN n'est pas une preuve d'optimisation, comme explicité infra. A contrario, ne pas envoyer de données à l'IRSN ne signifie pas nécessairement que les pratiques ne sont pas évaluées et optimisées.

Il est ainsi possible, voire probable, que la participation des établissements pratiquant la radiologie conventionnelle soit sous-évaluée mais elle demeure néanmoins la plus faible des trois domaines concernés par les NRD. 


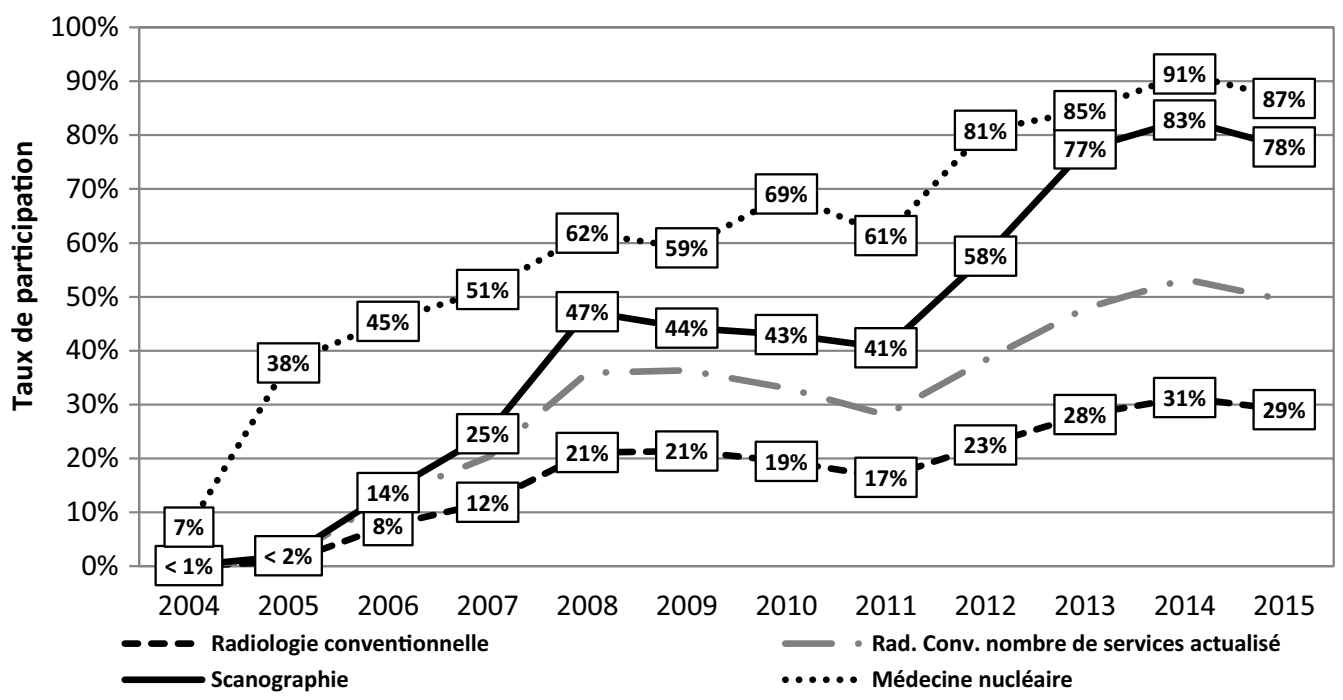

Fig. 1. Évolution de la participation annuelle des établissements français pratiquant des actes de radiologie conventionnelle, scanographie et médecine nucléaire entre 2004 et 2015.

Fig. 1. Evolution of the number of French departments performing radiology, computed tomography and nuclear medicine transmitting data between 2004 and 2015.

La disparité des pratiques et un cadre réglementaire moins strict conduisent ainsi à disposer d'une évaluation de la participation à la fois faible mais aussi entachée d'une incertitude importante en radiologie conventionnelle.

\section{De la formalité administrative à l'outil d'aide à l'optimisation...}

Dès lors qu'une démarche est imposée par un texte réglementaire, elle devient potentiellement ressentie comme négative et inutile, une corvée bureaucratique. Les NRD n'échappent pas à cet écueil.

Si la popularité n'est pas l'objectif visé par les NRD, cette perception a néanmoins des conséquences négatives car elle conduit parfois les professionnels concernés à occulter l'évaluation des doses qu'ils délivrent. Il arrive que des résultats significativement supérieurs aux NRD soient transmis et que les professionnels découvrent ce dépassement lorsque l'IRSN les contacte pour écarter toute erreur de saisie.

Lorsque la démarche n'est pas perçue comme bureaucratique, les professionnels appréhendent bien l'intérêt des NRD et de nombreux exemples de recueil de données NRD ayant conduit à détecter des dysfonctionnements de matériel ou des protocoles d'acquisition inadaptés pourraient être cités.

Depuis 2004, l'IRSN peinait à différencier le simple transfert de données - parfois sans aucune analyse qualitative - de l'envoi consécutif à une véritable évaluation et analyse des pratiques.

Dans son dernier bilan, l'IRSN a analysé l'évolution des résultats d'évaluations dosimétriques pour des établissements ayant transmis, dans un intervalle de 3 ans et pour une même installation, des données d'un même type d'examen (Roch et Célier, 2016; Roch et al., 2018). Il apparait que plus les relevés dosimétriques initiaux sont proches voire dépassent le NRD, plus le taux d'établissements ayant optimisé les pratiques est élevé, pour atteindre $100 \%$ lorsque le premier résultat dépasse le NRD. L'ampleur de la diminution de dose lors du 2 relevé est également proportionnelle à la valeur de dose initiale. L'intérêt des NRD comme initiateur d'actions d'optimisation, dans les établissements transmettant des données à l'IRSN, apparaît concrètement dans cette analyse et comble en partie le biais cité précédemment.

Il est par contre difficile d'évaluer le nombre d'établissements qui optimisent leur pratique mais ne transmettent pas de données à l'IRSN, attitude qui paraît toutefois paradoxale puisque la transmission est souvent un élément valorisant requis dans les démarches de certification, de développement professionnel continu ou d'évaluation des pratiques et d'assurance qualité (OGDPC, 2017; Labelix, 2017; HAS, 2012, 2014).

Il convient également de préciser le rôle des NRD dans le processus d'optimisation. Fréquemment présenté comme un «outil d'optimisation», les NRD sont plutôt, au travers du «repère» qu'ils fournissent aux professionnels, un outil informatif d'aide à l'optimisation. La comparaison des doses relevées par les professionnels aux valeurs de NRD leur donne une indication sur la nécessité de revoir leur pratique et de l'améliorer d'un point de vue dosimétrique. Les outils d'optimisation sont plutôt, à titre d'exemples, les dispositifs de modulation du courant dans le tube en scanographie ou la filtration additionnelle en radiologie.

C'est dans cet objectif d'améliorer l'aide à l'optimisation que l'IRSN a choisi - en accord avec les recommandations internationales les plus récentes - de compléter l'indicateur «d'alerte» représenté par le $75^{\mathrm{e}}$ centile, par une valeur-guide $\left(50^{\mathrm{e}}\right.$ centile) visant à encourager une continuité dans l'optimisation des doses (Roch et Célier, 2016).

\section{Une photographie des pratiques}

Nonobstant les incertitudes liées à la faiblesse de participation pour la radiologie conventionnelle, le dispositif actuel de recueil des NRD peut être considéré comme efficace 


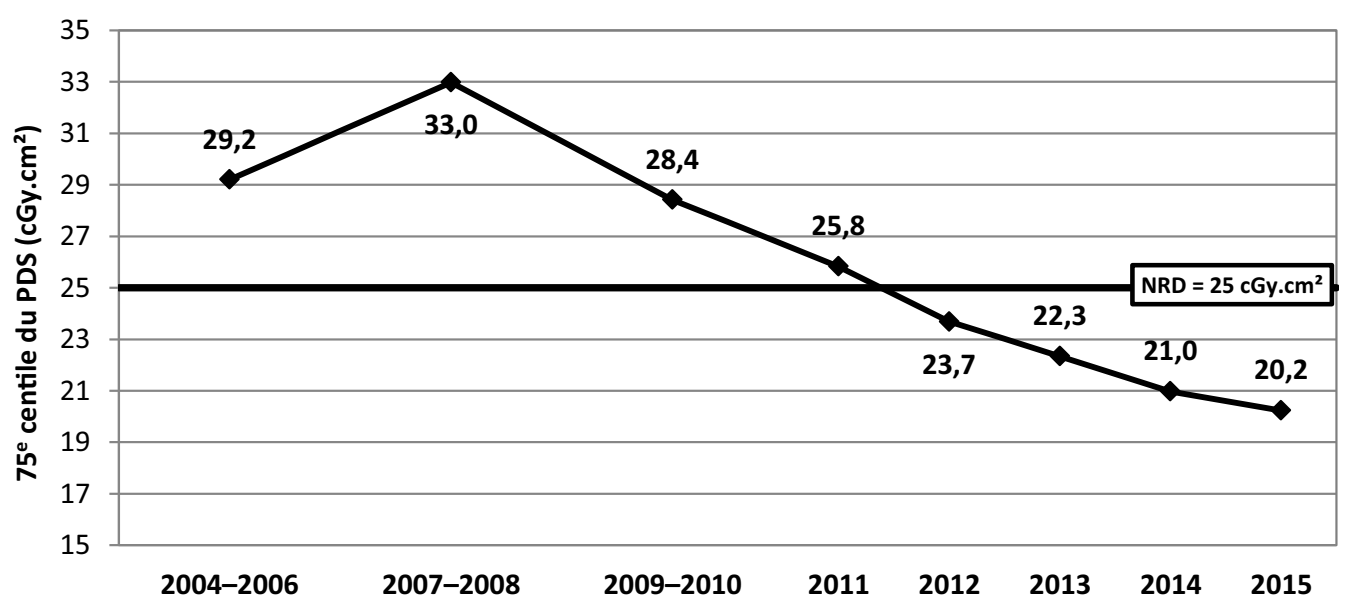

Fig. 2. Évolution du $75^{\mathrm{e}}$ centile du produit dose.surface (PDS) pour l'examen du thorax de face (postéro-antérieur) chez l'adulte entre 2004 et 2015.

Fig. 2. Evolution of the 75 th percentile value of the dose.area product (DAP) for the chest postero-anterior examination (adult) between 2004 and 2015 .

en termes de volume de données et de représentativité de la pratique nationale. Il est à noter qu'en matière de NRD, la France dispose d'un des systèmes les plus efficaces et dynamiques pour le recueil massif de données et le suivi des doses, compte-tenu du nombre d'établissements, d'installations et de patients pris en charge. De nombreux pays, y compris le Royaume-Uni qui dispose d'une grande expérience en matière de NRD, ont choisi de collecter leurs données NRD en s'appuyant sur des enquêtes périodiques ciblées sur un panel d'établissements d'imagerie limitant ainsi le nombre et l'origine des données recueillies ( $25 \%$ des services d'imagerie et $30 \%$ des scanners) (Hart et al., 2012; Shrimpton et al., 2014).

La mise en œuvre depuis 2011 d'un portail de télétransmission associé à une base de données, a particulièrement contribué au développement du dispositif et à l'amélioration de la qualité et du suivi des données collectées $(\text { IRSN })^{1}$. Des analyses selon des critères techniques tels que les paramètres d'exposition ou l'âge des appareils ont par exemple été rendues possibles.

Les résultats présentés successivement dans les cinq rapports de l'IRSN ont permis de mesurer l'évolution des doses depuis 2004, mais pas seulement. Les évolutions technologiques - capteur plan versus film en radiologie, reconstruction itérative en scanographie, temps de vol en tomographie par émission de positons en médecine nucléaire - et leur impact sur les doses font également partie des informations que le dispositif des NRD permet de mettre en exergue.

$\mathrm{Au}$ final, il apparaît que l'amélioration technologique induit une diminution des doses. Il est important de souligner que cela met en évidence la mise en œuvre d'une démarche d'optimisation au sein des établissements d'imagerie ayant bénéficié d'évolutions technologiques sur leurs appareils. L'optimisation est trop souvent directement imputée - parfois par les professionnels eux-mêmes - à la modernisation des appareils. Or, des équipements neufs, s'ils ne sont pas

\footnotetext{
1 Recueil et transmission des données NRD à l'IRSN. Accès le : 12/10/2017, depuis : http://nrd.irsn.fr/transmission.
}

correctement pris en main et si les protocoles ne sont pas adaptés, peuvent conduire à des expositions supérieures à celles engendrées par des systèmes plus anciens (Mafalanka et al., 2014, 2015; Roch et al., 2017, 2018).

Comme le montrent les résultats de la dernière analyse des données NRD, avec des valeurs de NRD qui peuvent être revues à la baisse de $40 \%$ en moyenne en radiologie, $35 \%$ en scanographie et $8 \%$ en médecine nucléaire par rapport à 2011 , date de la dernière mise à jour réglementaire, force est de constater qu'en France les pratiques professionnelles sont en constante amélioration du point de vue dosimétrique. À titre d'exemple, la radiographie du thorax de face, examen le plus fréquemment transmis à l'IRSN, illustre l'amélioration des pratiques depuis la mise en œuvre des NRD en France (Fig. 2) avec un produit dose.surface (PDS) qui a diminué de près de $40 \%$ depuis 2008 et qui est inférieur d'environ $20 \%$ à la valeur du NRD correspondant.

\section{Les faiblesses}

En 2015, un groupe de travail (GT) issu du Groupe Permanent d'Experts pour la radioprotection dans les applications médicales et médico-légales des rayonnements ionisants (GPMED) a dressé un bilan de la mise en œuvre des NRD en France incluant une comparaison avec d'autres pays européens. À l'issue de ce travail, le GT a proposé des actions de nature à améliorer la participation des centres d'imagerie au recueil et à l'analyse des données dosimétriques en lien avec les NRD ainsi que des évolutions des dispositions réglementaires (ASN, 2015 ; GPMED, 2015). Les treize propositions formulées visent à compenser les principaux écueils du dispositif actuel du point de vue organisationnel et réglementaire.

Les axes d'amélioration mentionnés ci-après concernent plus particulièrement des éléments issus du retour d'expérience constitué par les treize années de recueil et d'analyse des données par l'IRSN.

Le premier concerne l'imagerie médicale pédiatrique. Même si la proportion des examens d'imagerie réalisés chez l'enfant est faible (environ $5 \%$ des actes) comparativement à 
l'adulte, la radiosensibilité et l'espérance de vie des enfants en font une population qui mérite une attention toute particulière.

Des NRD pédiatriques ont été définis dès 2004 en radiologie. En 2011, ont été ajoutés des NRD pour des examens de scanographie et de médecine nucléaire chez l'enfant. Néanmoins, le système actuel est totalement inefficace en matière de recueil de données. Ce n'est pas une particularité française, car tous les pays ayant mis en œuvre les NRD sont confrontés à cette difficulté (ESR, 2015). Plusieurs causes sont principalement à l'origine de cette situation :

- le nombre d'actes chez l'enfant est faible dans la plupart des établissements d'imagerie, ceux-ci étant majoritairement réalisés dans quelques services spécialisés;

- la dose étant directement corrélée à la morphologie du patient, les données pédiatriques doivent être triées selon l'âge (le poids) des enfants;

- les professionnels ont la possibilité de choisir s'ils collectent des données adultes ou pédiatriques.

Pour les raisons citées ci-dessus, les professionnels fournissent préférentiellement les données des examens chez l'adulte. Au total, le nombre de données disponibles est très faible et ne permet pas de produire des analyses fiables.

Au cours de l'année 2016, l'IRSN a réalisé, en collaboration avec la Société française de radiologie (SFR), la société française de médecine nucléaire (SFMN), la Société française d'imagerie pédiatrique (SFIPP) et la Société française de physique médicale (SFPM), des études ciblées visant à collecter des données dosimétriques chez l'enfant auprès d'établissements spécialisés (Célier et al., 2017a, 2017b). Ces études vont permettre de proposer des NRD pédiatriques basés sur des données récentes et issues de pratiques réalistes.

La deuxième voie d'évolution des NRD actuels concerne le domaine de la pratique interventionnelle radioguidée. Dans le champ de la radiologie, de la scanographie et de la médecine nucléaire, les NRD visent à réduire la probabilité d'effets toxiques des rayonnements ionisants à long terme. En pratique interventionnelle radioguidée, alors qu'un risque d'effet déterministe est avéré et significatif, aucun niveau de référence (NR) n'est disponible (Etard et al., 2012b). En accord avec les exigences européennes récentes, la France projette de définir des NR dans ce domaine. Elle s'appuie en particulier sur des études françaises menées très récemment (Etard et al., 2017; Georges et al., 2017).

En troisième lieu, les modalités d'imagerie ont beaucoup évolué et de nouvelles sources d'exposition sont apparues. C'est le cas de l'imagerie hybride en médecine nucléaire. Les NRD existent actuellement pour la partie scintigraphique mais pas pour la partie scanographique qui représente pourtant la principale source d'exposition des patients lors de ce type d'examen. La définition prochaine d'un NRD pour la partie scanographique de la tomographie par émission de positons couplée à un scanner - examen le plus fréquent et le mieux «protocolisé »- devrait combler en partie ce retard (Etard et al., 2012a).

Ensuite, et là encore la France ne fait pas exception, les NRD sont trop focalisés sur la dose et ne prennent pas en compte de façon qualitative la performance diagnostique de l'examen au travers de la qualité d'image. Sur ce point, la difficulté à évaluer objectivement cette grandeur avec des outils simples et standardisés est un obstacle à l'introduction d'un critère de qualité d'image, associé au critère dosimétrique, dans les NRD. Des propositions existent et des travaux sont en cours pour pallier cette faiblesse qui limite l'efficacité des NRD mais sa mise en œuvre en France nécessitera obligatoirement une uniformisation des pratiques et une forte implication des radiologues et médecins nucléaires, au travers notamment de leurs sociétés savantes, seules aptes à définir des critères de qualité image corrélés à la performance des examens (Rehani, 2015; Verdun et al., 2015).

Enfin, les NRD, trop généralistes, ont montré leurs limites dans le domaine de la scanographie du fait de leur définition par région anatomique (thorax, abdomen...). En effet, pour une même région anatomique, divers protocoles correspondant à différentes qualités d'images recherchées et donc générant des doses variables peuvent être mis en œuvre selon l'objectif clinique visé. Des travaux sont actuellement en cours en France au sein d'un groupe de travail de la Société française de physique médicale (SFPM) et au niveau Européen dans le cadre d'un projet initié par la Commission européenne et piloté par l'European Society of Radiology (ESR) (ESR, 2017). Ces travaux visent à définir des NRD en scanographie par indication clinique.

\section{Conclusion et perspectives}

Le dispositif des NRD en France est confronté à un paradoxe. Perçu par certains comme trop complexe, rigide et rebutant par sa dimension réglementaire, d'autres le considèrent comme trop simpliste et insuffisamment ambitieux en termes d'optimisation. Le dispositif NRD semble donc être arrivé à un point d'équilibre et remplit globalement bien sa mission. Par ailleurs, le système apparaît comme efficace pour évaluer les pratiques au niveau national, il est reconnu au niveau international et il est adopté par les professionnels convaincus.

Il est donc nécessaire de mener de front des actions de formation et d'information, avec l'implication des sociétés savantes, pour convaincre et encourager les professionnels à s'appuyer sur les NRD pour évaluer et optimiser leurs pratiques et, en même temps, il faut poursuivre le développement du dispositif en tenant compte des évolutions des techniques et des pratiques, ainsi que des attentes des professionnels.

Dans les mois à venir, la réglementation des NRD va évoluer en s'appuyant sur les exigences de la directive Euratom 2013/59, les travaux de l'IRSN, les résultats des études spécifiques menées en France et sur les recommandations issues des nombreux travaux menés au niveau national et international (ASN, 2015; GPMED, 2015; IAEA, 2016a, 2016 b; Vano et al., 2018).

\section{Références}

ASN. 2015. Avis du GPMED sur les niveaux de référence diagnostiques en imagerie. Accès le: 12/10/2017, depuis: https://www.asn.fr/ Media/Files/00-GP/GPMED-2015-05-26-Avis.

ASN. 2017a. Demande d'autorisation de détention et d'utilisation de sources scellées et non scellées pour une activité de médecine 
nucléaire et/ou de biologie médicale incluant la recherche biomédicale (Formulaire AUTO/MED/MEDNU). Accès le: 05/ 10/2017, depuis: https://www.asn.fr/Professionnels/Activitesmedicales/Medecine-nucleaire/Formulaires.

ASN. 2017b. Demande d'autorisation de détention et/ou d'utilisation de scanner(s) à usage médical (Formulaire AUTO/MED/SCAN). Accès le : 05/10/2017, depuis : https://www.asn.fr/Professionnels/ Activites-medicales/Radiologie-et-scanographie/Formulaires.

ASN. 2017c. Déclaration de détention/utilisation d'appareils électriques générant des rayons $\mathrm{X}$ (Formulaire DEC/GX). Accès le : 05/10/2017, depuis : https://www.asn.fr/Professionnels/Activitesmedicales/Radiologie-et-scanographie/Formulaires.

CE. 1997. Directive 97/43/Euratom du Conseil du 30 juin 1997 relative à la protection sanitaire des personnes contre les dangers des rayonnements ionisants lors d'expositions à des fins médicales, remplaçant la directive 84/466/Euratom CE. Journal officiel de l'Union européenne.

CE. 2014. Directive 2013/59/Euratom du Conseil du 5 décembre 2013 fixant les normes de base relatives à la protection sanitaire contre les dangers résultant de l'exposition aux rayonnements ionisants et abrogeant les directives 89/618/Euratom, 90/641/ Euratom, 96/29/Euratom, 97/43/Euratom et 2003/122/Euratom CE. Journal officiel de l'Union européenne.

Célier D et al. 2017a. Mise à jour des NRD en pédiatrie : enquête SFMN-SFPM-IRSN sur les pratiques, Méd. Nucl. 41(3): 1.

Célier D et al. 2017b. Optimisation des doses délivrées en radiologie et scanographie pédiatriques: enquête SFIPP-SFR-IRSN sur les pratiques. Accès le : 12/10/2017, depuis : http://www.sfip-radiopedia trie.org/index.php/congres/resumes-congres-sfipp-2/sfipp-toulouse16/712-c01-optimisation-des-doses-delivrees-en-radiologie-et-scano graphie-pediatriques-enquete-sfipp-sfr-irsn-sur-les-pratiques-

Cour des comptes. 2016. L'imagerie médicale. Accès le : 12/10/2017, depuis : www.ccomptes.fr.

ESR. 2015. PiDRL Project. Accès le: 12/10/2017, depuis : http:// www.eurosafeimaging.org/pidrl/.

ESR. 2017. EUCLID project. Accès le : 26/09/2017, depuis : http:// www.eurosafeimaging.org/euclid/about.

Etard C, Aubert B. 2014. Exposition de la population française aux rayonnements ionisants liée aux actes de diagnostic médical en 2012. (Rapport PRP-HOM/2014-6).

Etard C et al. 2012a. National survey of patient doses from wholebody FDG PET-CT examinations in France in 2011, Radiat. Prot. Dosim. 152(4): 334-338.

Etard C et al. 2012b. Need for improving patient radiation protection in interventional radiology-A review of recent overexposure cases in France. Radiation Protection in Medicine-Setting the Scene for the Next Decade (IAEA, Bonn).

Etard C et al. 2012c. French population exposure to ionizing radiation from diagnostic medical procedures in 2007, Health Phys. 102(6): 670-679.

Etard C et al. 2017. Patient dose in interventional radiology: a multicentre study of the most frequent procedures in France, Eur. Radiol. 27(10): 4281-4290.

Georges JL et al. 2017. Radiation doses to patients in interventional coronary procedures - Estimation of updated national reference levels by dose audit, Radiat. Prot. Dosim. 175(1):17-25.

GPMED. 2015. Recommandations sur les niveaux de référence diagnostiques en imagerie médicale. Accès le: 12/10/2017, depuis : http://www.asn.fr/Informer/Actualites/Niveaux-de-refe rence-diagnostiques-en-imagerie-medicale.

Hart D et al. 2012. Doses to patients from radiographic and fluoroscopic X-Ray imaging procedures in the UK-2010 review. (HPA-CRCE-034).
HAS. 2012. Radioprotection du patient et analyse des pratiques DPC et certification des établissements de santé-Guide méthodologique. Accès le: 12/10/2017, depuis : https://www.has-sante.fr/ portail/upload/docs/application/pdf/2013-03/radioprotection_du_ patient_et_analyse_des_pratiques_dpc_et_certification_des_eta blissements de sante guide.pdf.

HAS. 2014. Manuel de certification des établissements de santé V2010. ACC01-T052-E, 2014. Accès le: 12/10/2017, depuis : http://www.has-sante.fr/portail/upload/docs/application/pdf/ 2014-03/manuel_v2010_janvier2014.pdf.

IAEA. 1996 rev. 2014. Radiation Protection and Safety of Radiation Sources: International basic safety standards. IAEA Safety Standards Series No. GSR Part 3.

IAEA. 2016a. Safety report project: Patient Dose Monitoring and the Use of Diagnostic Reference Levels for the Optimization of Protection in Medical Imaging. Accès le : 27/06/2017, depuis : https://rpop.iaea.org/RPOP/RPoP/Content/News/6-tm-patientdose-monitoring.html.

IAEA. 2016b. Technical Meeting on Patient Dose Monitoring and the Use of Diagnostic Reference Levels for the Optimization of Protection in Medical Imaging. Accès le: 19/05/2017, depuis : https://rpop.iaea.org/rpop/rpop/Content/News/6-tm-patient-dosemonitoring.htm.

ICRP. 1991. 1990 Recommendations of the International Commission on Radiological Protection, Ann ICRP 21(1-3): 1-201.

ICRP. 1996. Radiological protection and safety in medicine. A report of the International Commission on Radiological Protection, Ann ICRP 26(2): 1-47.

JORF. 2004. Arrêté du 12 février 2004 relatif aux niveaux de référence diagnostiques en radiologie et en médecine nucléaire. Journal officiel de la République Française du 16 mars.

JORF. 2012. Arrêté du 24 octobre 2011 relatif aux niveaux de référence diagnostiques en radiologie et en médecine nucléaire. Journal Officiel de la République Française du 14 janvier.

Labelix. 2017. Référentiel qualité en Imagerie Médicale. Accès le : 04/10/2017, depuis : http://www.labelix.org/.

Mafalanka FC et al. 2014. Dose optimisation in coronary CT angiography: is it restricted to the prospective ECG gating mode? Results from a multicentre study, in ECR 2014, Vienna.

Mafalanka FC et al. 2015. Establishment of diagnostic reference levels in cardiac CT in France: a need for patient dose optimisation, Radiat. Prot. Dosim. 164(1-2): 116-119.

OGDPC. 2017. Organisme Gestionnaire du Développement Professionnel Continu. Accès le : 04/10/2017, depuis : https://www. mondpc.fr/.

Rehani MM. 2015. Limitations of diagnostic reference level (DRL) and introduction of acceptable quality dose (AQD), Br. J. Radiol. 88(1045): 20140344.

Roch P. 2010. Analyse des données relatives à la mise à jour des niveaux de référence diagnostiques en radiologie et en médecine nucléaire. Bilan 2007-2008. (Rapport DRPH 2010-15).

Roch P, Aubert B. 2013. French diagnostic reference levels in diagnostic radiology, computed tomography and nuclear medicine: 2004-2008 review, Radiat. Prot. Dosim. 154(1): $52-75$.

Roch P, Célier D. 2012. Analyse des données relatives à la mise à jour des niveaux de référence diagnostiques en radiologie et en médecine nucléaire. Bilan 2009-2010. (Rapport PRP-HOM/ 2012-12).

Roch P, Célier D. 2014. Analyse des données relatives à la mise à jour des niveaux de référence diagnostiques en radiologie et en 
médecine nucléaire. Bilan 2011-2012. (Rapport PRP-HOM/ 2014-9).

Roch P, Célier D. 2016. Analyse des données relatives à la mise à jour des niveaux de référence diagnostiques en radiologie et en médecine nucléaire. Bilan 2013-2015. (Rapport PRP-HOM 2016-00006).

Roch P et al. 2017. Patient exposure from nuclear medicine in France: national follow-up and influence of the technology through diagnostic reference levels data analysis, Radiat. Prot. Dosim. Oct(13): 1-8.

Roch P et al. 2018. Using Diagnostic Reference Levels to evaluate the improvement of patient dose optimisation and the influence of recent technologies in radiography and computed tomography, Eur. J. Radiol. Jan(98): 68-74.

Shrimpton PC et al. 2014. Doses from Computed Tomography (CT) Examinations in the UK-2011 Review. (PHE-CRCE-013).

Talbot A. 2008. Analyse des données relatives à la mise à jour des niveaux de référence diagnostiques en radiologie et en médecine nucléaire. Bilan 2004-2006. (Rapport DRPH 2008-02).

Vano E et al. 2018. Diagnostic Reference Levels in Medical Imaging. ICRP Publication, Ann. ICRP.

Verdun FR et al. 2015. Image quality in CT: From physical measurements to model observers, Phys. Med. 31(8): 823-843.

Citation de l'article : Roch P, Célier D, Dessaud C, Etard C. 2018. Les niveaux de référence diagnostiques en France: une perception contrastée face à un dispositif perfectible mais efficace. Radioprotection 53(1): 13-19 